\title{
Geospatial Information Service Based on Ad Hoc Network
}

\author{
Yun ZHANG, Fuling BIAN \\ International School of Software, Research Center of Spatial Information and Digital Engineering, \\ Wuhan University, Wuhan, China \\ Email: zhangyun604@yahoo.com.cn \\ Received January 16, 2009; revised February 10, 2009; accepted February 18, 2009
}

\begin{abstract}
The mobile geospatial information service involves the domain of mobile communication, mobile computing, geospatial information service and other techniques. This paper focuses on the integration of spatial information and mobile communication technologies. The author proposes the architecture of mobile geospatial information service based on the Ad Hoc network. On the basis of this architecture, a system is developed, and applied in correlative fields.
\end{abstract}

Keywords: Spatial Data, Ad Hoc Network, Geospatial Information Service

\section{Introduction}

With the development of electronic technology, especially in the fields of computer and internet technology, mobile communication has made a rapid progress in recent years. More and more mobile phones with powerful functions have emerged in our daily life. At the same time, cell phone users are not satisfied with the only function of call service while more and more mobile services have emerged. So the proportion of mobile services rate in the communications revenue has increased year by year.

According to statistics in the resource management, socio-economic activities and public life, more than 80 percent of information involves in the geographic information with spatial localization feature. The fast development of technological and subject matter has got much attention in recent years, which are used as an important means of communication for acquiring, arranging, analyzing and managing geospatial data in productive activities and Geographic Information System. Meanwhile, the users are not satisfied with acquiring GIS Services in the use of computer equipments and Geographic Information System. Nowadays, computer network and wireless communication technology have become so widespread. It offers a new direction in the development of GIS.

\section{Mobile Geographic Information Service}

Geographic Information System (GIS for short) is a kind of technology for saving and analyzing spatial information, integrated with computer graphics and data. GIS is the spatial information system that has been compiled in purpose of specific application. Under the help of computer hardware, software facilities and network, it is user for collect, input, store, process, display, update and provide kinds of dynamic geographic information application, with the method of geographic models [1].

GIS has passed through two great changes: from the stage of GISystem to GIScience, then transferred to the stage of GIService [2,3]. In modern society, network technology and wireless communication technology are developing rapidly. With the development of GIS and the two technologies, a new spatial information service and application model was born, i.e. (Mobile Geographic Information Service, MGIS) [4]. MGIS offers mobile sharing with geographic information in the integration of computer, telecommunication and $3 \mathrm{~S}$ technologies. It can help the users to acquire the maximum amount of information in a finite-range space. At the same time, it truly meets the users' needs in geography information query and decision making. It is an important content of GIService research that how to make users satisfied with geographic information conveniently geo-information for anyone and anything at anywhere and anytime [5]. 
Nowadays, Internet-centered information network has basically satisfied the need for information in anytime. However, Internet builds on fixed cable network failed to meet the need for acquiring the information services in mobile.

In the research of mobile geospatial information service, we should take such factors into account as:

1) The limited bandwidth and frequently disconnection in wireless networks

The disconnection and transmission delay don't need consideration in geospatial information service in traditional networks. The delay, bandwidth, signal intensity and communication protocol may be changing in the wireless networks. As a result, mobile geospatial information service must be fit for the technique status in the face of communication field in modern time.

2) Enhancement adaptability of service providing methods

Compare to cable network, wireless network has a more flexible deployment. It has many different ways in which can be connected to the network: from Infrared Ray to Bluetooth within a few-mile limit, from WLAN to satellite relay and other communication modes covering the whole world. All these factors can be considered in application system design. What's more, the cable can be used as an auxiliary method.

3) Low security in data transmission

Mobile geospatial information service applies electromagnetic wave to send or receive data through the air. It can work without cable and other transmission media. But we have to note that the process is easily perturbed by external environment because of transmission media's vulnerability. Meanwhile, the data transferred will be stolen by illegal users for uncertainty in the transmission process. Therefore, we should try to avoid long distance wireless transmission in the architecture design.

4) Limited energy and resources of mobile terminal

The mobile terminal is supplied from storage batteries. Due to the requirement of power supply, mobile equipment can not work for a long time. On one hand, the resource in mobile terminal would differ from fixed hosts in the increase of portability. On the other hand, it has a limited computational power. As a result, rational allocation of the data is necessary, especially between mobile terminal and fixed hosts. In this way, it can enhance the rates of resource utilization efficiency in querying, processing and store management.

5) Multiplicity in architecture design

Traditional systems are mostly based on Client/Server mode, as indicated in Figure 1. While in mobile environment, dynamic computing resource and network joint nodes are full of uncertainty. The mobile terminal is of versatile features and configuration. According to difference between wireless networks connectivity and the type and capability of mobile terminal equipment, mo- bile geospatial information service should design different architecture to achieve the full potential of hardware and networks [6].

The basic network configuration of mobile geospatial information service and application environment have experienced great changes for traditional geospatial information service. Although wireless network is the similar as the extension of wired network, there is a big difference between them both in technologies and system design. So research on mobile geospatial information service must make a concrete analysis for these features.

\section{Ad Hoc Network}

Wireless network offers the users the data exchange and acquisition in anytime and any place. And it has the function of keeping connecting in moving state. In general, mobile communication network has a center. It only works in presupposition based network establishment. In some special occasions, such as on the battlefields, the rescue attempt after flood and earthquake, field operations and so on, traditional centered cellular networks can not meet the requirements of communication. In this way, a new network technology-Ad Hoc network has emerged, which need not rely on fixed telecommunication.

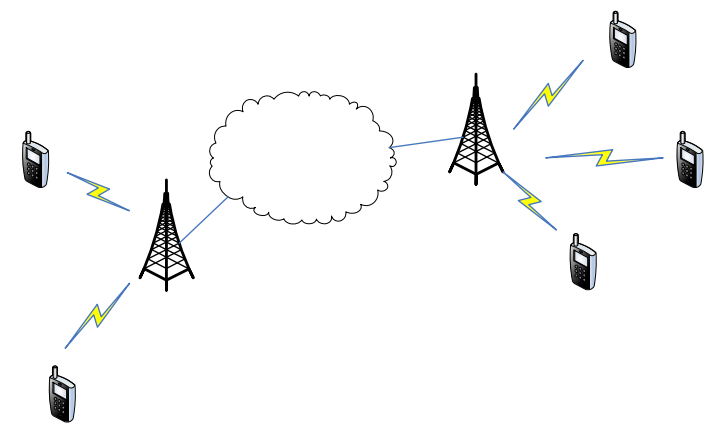

(a) Cellular telecommunication network.

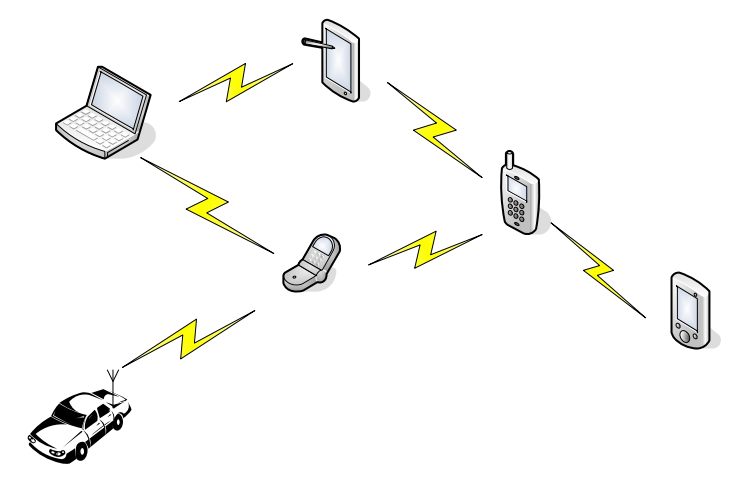

(b) Ad hoc network.

Figure 1. The comparison of cellular telecommunication network and ad hoc network. 
Ad Hoc network is made up of a group of wireless communication and connecting network module, without any fundamental establishment. When the cable network can not work in order, like on the battlefield and in the task of emergency relief, Ad Hoc network offers a feasible technology of communication and information storage. When two mobile terminals are in each other's covering range, they can communicate directly in Ad Hoc network [7]. But the communication coverage of mobile terminal is limited. The two hosts which are far away from each other can be linked by other nodes' forwarding.

In Ad Hoc network, the moving node changes the topology of network. Routing in ad hoc wireless networks has to cope with specifics such as limited bandwidth, high error rates, dynamic topology, resource poor devices, power constraints and hidden and exposed terminal problems. It is the essential part for Ad Hoc network that how to choose the routes to destination accurately and quickly. The typical routing algorithms are as follows [8].

DSDV (Destination-Sequenced Distance-Vector) is a Table driven routing protocol based on the classical Bellman-Ford routing algorithm. by improving freedom from loops in routing tables by using sequence numbers.
DSR (Dynamic Source Routing) generates routes only when desired. When a packet needs to be sent to a destination whose route doesn't exist, a route discovery process is initiated. If the route has been existed, a route maintenance procedure is invoked. AODV (Ad hoc On-demand Distance Vector Routing) is a pure on-demand routing protocol. It is based on DSDV protocol.

\section{Geospatial Information Service Architec- ture Based on Ad Hoc Network}

In wireless environment of geographic information service, spatial data transmission focuses on wireless network and mobile equipments in different environments.

The transmission scale of wireless local area network is relatively small. If we want to enlarge the transmission coverage, we can apply the method of adding antennas. The user end can work in the mode of Ad Hoc if a user wants to set up his own network without the help of access points. As for the problem faced by mobile geographic information service, it is necessary to apply Ad Hoc network into the architecture, as indicated in Figure 1.

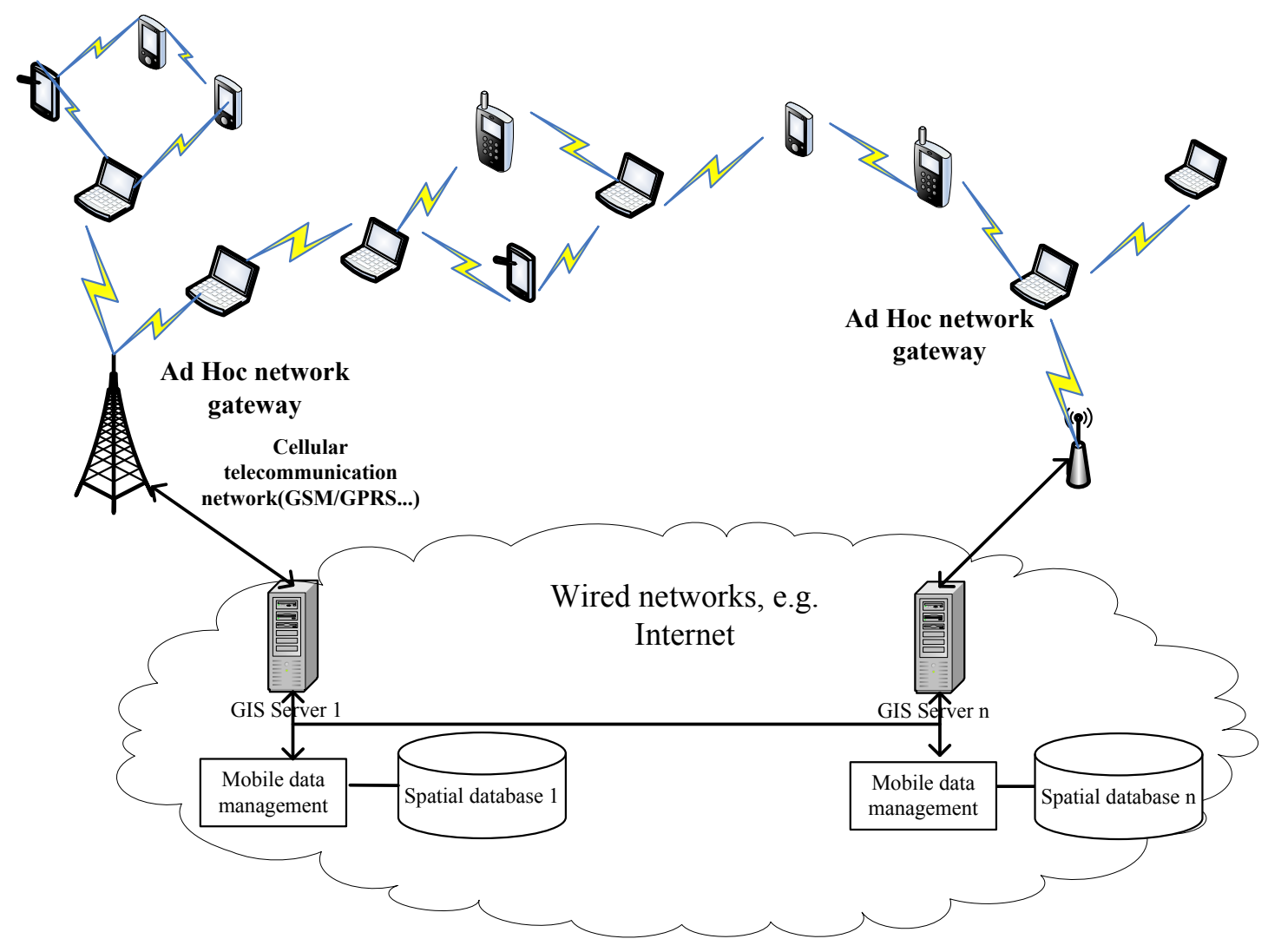

Figure 2. Geospatial information service architecture based on ad hoc network. 
As indicated in Figure 1, geospatial information service is made up of mobile terminal, mobile unit, mobile supporting station, fixed host and wired networks.

The data transmission among mobile nodes in Ad Hoc network adopts the advanced technology of distributed computing. It can break the limits of transmission distance in traditional WLAN. High speed wired network forms the connecting backbone with fixed nodes including file server, communications server, file server and database service. Wireless data transmission by mobile terminals with the Ad-Hoc routing mode is an effective method to realize wireless GIS. With the help of this technology, it can offer a network linked by many wireless node in short distance, the nodes in large scale can also be linked by multi-hop in nearby nodes to build a large Ad hoc network. In addition, traditional wireless Ad Hoc routing protocols needs to be optimized in application to mobile geospatial information service.

First the mobile terminal needs to find the Ad Hoc network gateway to connect with the cellular telecommunication network or Internet. The network gateway periodically transmits package all around to broadcast own existence. If the mobile terminal is within one-hop distance from the gateway the, the mobile terminal may find the mobile gateway through the received broadcast information. If the distance is beyond one hop, the mobile terminal will send the control package to seek the gateway, then this mobile terminal will receive from one or more than one reply from the Ad Hoc network gateway. The mobile terminal will select the gateway with least hop number to connect with the cellular telecommunication network or Internet. By using the gateway router, several Ad Hoc network may be interconnected to enable the mobile node to reach Internet.

\section{The Experimental System}

Based on the above architecture, the author carries out a platform of urban public administration and service information management. It is designed to gather the urban management related data, position the accurate location of emergencies, carry on the effective dispatch promptly, direct the related personnel to process the accident. The client interface on intelligent mobile phone is indicated as in Figure 3.

The success of experimental system proves that the spatial data transmission mechanism among the mobile terminals by Ad Hoc network proposed in our paper is feasible. From the angle of experiment, it is feasible to prove the rationality of this architecture. It not only can save the resources of cellular telecommunication system, but also overcome the limit of geographic information service implementation created by mobile environment.
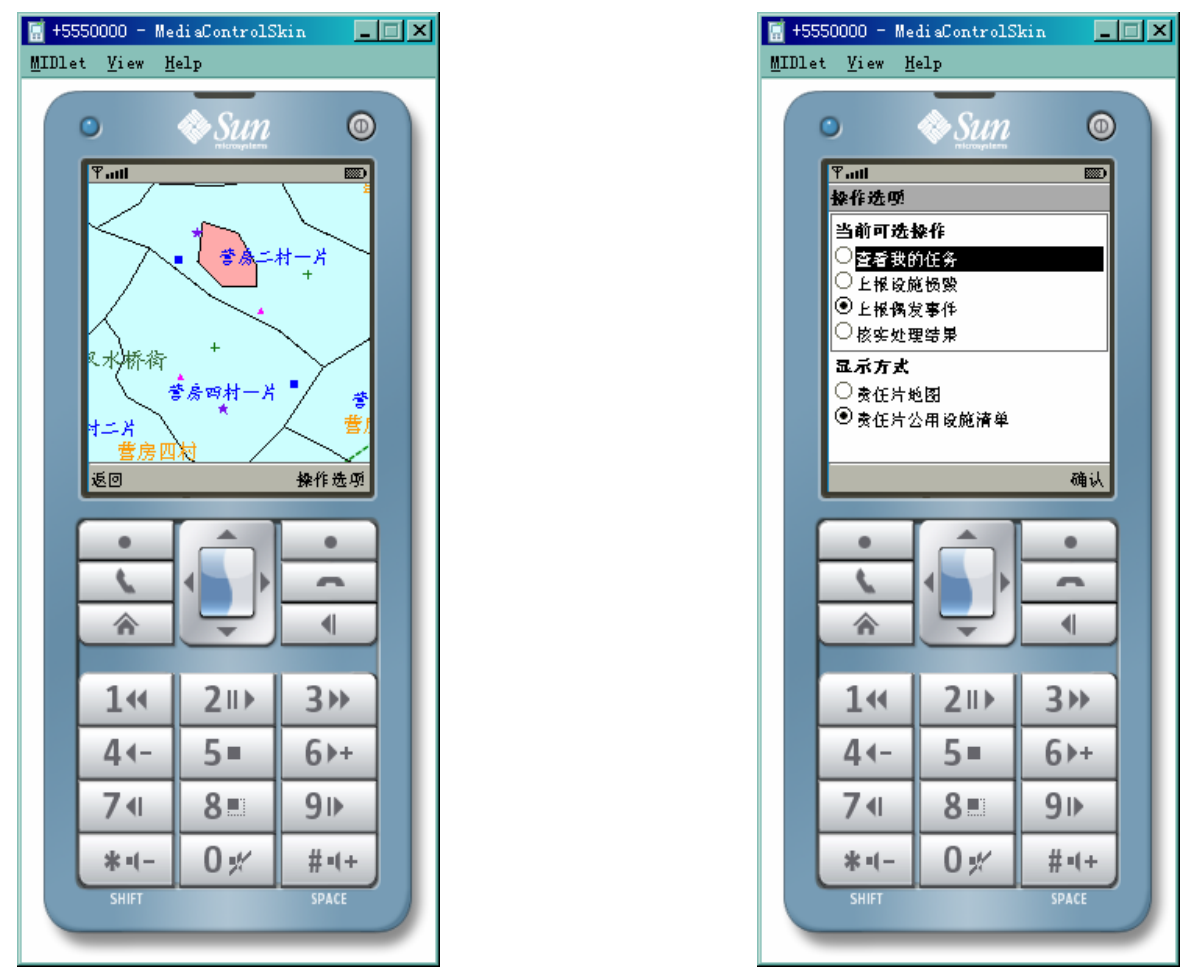

Figure 3. The client interface on intelligent mobile phone. 


\section{Conclusions}

The emphasis of the research on mobile geospatial information service is how to organize and transmit the kinds of geographic information with less network resource and higher transmission efficiency. This paper explores the geospatial information service based on Ad Hoc network aiming to realize the geographic data sharing among mobile terminals, furtherly to increase the data safety and quality in large-scale wireless data transmission process. But there are both advantages and disadvantages on kinds of protocols in network performance. We need to further our research on how to optimize the protocols to obtain the better network performance according to the features of mobile geospatial information service.

\section{References}

[1] S. P. Chen, "Urbanization and urban GIS," Science Press, 2001.

[2] J. W. Shi, K. W. Kwan, J. N. Cao, et al., "A proactive approach for mobile GIS [C]," Proceedings of IEEE 58th Vehicular Technology Conference, Vol. 2, pp. 1000-1004, 2003.

[3] H. Wen, "Design and implementation of a prototype mobile GIS based on J2EE/J2ME," Beijing, Peking University, 2005.

[4] C. H. Peng, Y. F. Liu, L. Yan, J. Y. Liu, and J. H. Zheng, "Research on key techniques of Java-based mobile geographic information service," Computer Engineering and Applications, No. 11, 2007.

[5] D. R. Li, "The development of RS and GIS in the 21st century," Geomatics and Information Science of Wuhan University, No.2, pp. 127-131, 2003.

[6] M. Xu, "Mobile computing technology," Tsinghua University Press, September 2008.

[7] S. R. Zheng and H. T. Wang, "Ad hoc network technology," Post and Telecom Press, October 2005.

[8] K. U. R. Khan, R. U. Zaman, and A. V. Reddy, "Performance comparison of on-demand and table driven ad hoc routing protocols using NCTUns," Proceedings- UKSim 10th International Conference on Computer Modelling and Simulation, EUROSIM/UKSim2008. 